\title{
Overexpression of Pin 1 and rho signaling partners correlates with metastatic behavior and poor recurrence-free survival of hepatocellular carcinoma patients
}

\author{
Lui $\mathrm{Ng}^{1}$, Virginia Kwan ${ }^{1}$, Ariel Chow ${ }^{1,2}$, Thomas Chung-Cheung Yau ${ }^{3}$, Ronnie Tung-Ping Poon ${ }^{1,2}$,
}

Roberta Pang ${ }^{1,2^{*}}$ and Wai-Lun Law ${ }^{1}$

\begin{abstract}
Background: Identification of molecular markers for early detection or prediction of metastasis is crucial for both management of HCC patient postoperative treatment and identify new therapeutic targets to inhibit HCC progression and metastasis. In the current study, we investigated the clinical correlation between Pin1, RhoA and RhoC and their association with HCC metastasis.

Methods: Using a randomized study design of primary HCC samples from 139 patients, we determined messenger RNA expression of Pin1, RhoA and RhoC and their prognostic value.

Results: Our findings demonstrated for the first time the clinical correlation of Pin1 in HCC metastasis. Pin1, RhoA and RhoC transcript levels were significantly higher in HCC specimens when compared with the paired adjacent non-tumorous liver. Pin1 overexpression was closely correlated with that of RhoA $(R=0.562, p<0.001)$ and RhoC $(R=0.529, p<0.001)$, and their co-overexpressions correlated with metastatic HCC $(p=0.000012)$ and poor recurrence-free survival of HCC patients $(p<0.00001$ ), which showed better prognostic significance than either Pin1, RhoA or RhoC overexpression alone. Co-overexpressions of Pin $1+$ RhoA/RhoC were also an independent factor for predicting development of metastasis after curative resection in our multivariate regression model $(p<0.001)$.
\end{abstract}

Conclusion: Pin1, RhoA and RhoC co-overexpressions are prognostic factor for metastatic HCC and predict poor recurrence-free survival.

Keywords: Pin 1, RhoA, RhoC, Metastasis

\section{Background}

Hepatocellular carcinoma (HCC) is a leading cause of cancer mortality worldwide, especially in Eastern Asian regions including Hong Kong and China where hepatitis $\mathrm{B}$ is more prevalent, while cases of $\mathrm{HCC}$ are also slightly increasing in low-incidence areas such as the United States and Canada [1]. Despite recent advances in therapeutic strategy and surgical techniques in treatment of primary cancer, the prognosis of HCC patients is still

\footnotetext{
* Correspondence: lui_ng@hotmail.com; robertap@hku.hk

'Department of Surgery, Li Ka Shing Faculty of Medicine, The University of Hong Kong, Pok Fu Lam, Hong Kong

${ }^{2}$ Centre for Cancer Research, Li Ka Shing Faculty of Medicine, The University of Hong Kong, Pok Fu Lam, Hong Kong

Full list of author information is available at the end of the article
}

poor due to a high incidence of postoperative metastasis and recurrence [2]. Increasing researches have been focused on investigating the molecular pathways leading to cancer metastasis and the fundamental steps such as cell invasion and migration, in order to discover molecular markers for early detection or prediction of metastasis which is crucial for management of patient postoperative treatment, as well as to identify novel therapeutic targets to inhibit HCC progression and metastasis.

The identification and characterization of a peptidylprolyl cis/trans isomerase, Pin1, has led to the discovery of a new postphosphorylation regulatory mechanism in cell signaling pathways through promoting the cis-trans isomerization of specific proteins that are phosphorylated at

(c) The Author(s). 2019 Open Access This article is distributed under the terms of the Creative Commons Attribution 4.0 International License (http://creativecommons.org/licenses/by/4.0/), which permits unrestricted use, distribution, and reproduction in any medium, provided you give appropriate credit to the original author(s) and the source, provide a link to the Creative Commons license, and indicate if changes were made. The Creative Commons Public Domain Dedication waiver (http://creativecommons.org/publicdomain/zero/1.0/) applies to the data made available in this article, unless otherwise stated. 
Ser/Thr-Pro motifs [3-5]. Such conformational changes can have profound effects on the function of many Pin1 substrates. By this, Pin1 plays an important role in many cellular events, such as cell cycle progression, cell proliferation, and transcriptional regulation. In $\mathrm{HCC}$, we have previously reported a high prevalence of Pin 1 over-expression [6]. Our group and the others demonstrated that through interaction with different substrates, Pin 1 induced cell proliferation, enhances cellular transformation and inhibited apoptosis in HCC [6-10].

The aim of this study was to investigate the clinical correlation between Pin1 and HCC metastasis. We also examined the potential interaction between Pin1 and two members of the Rho subfamily of the Ras superfamily of homologious genes, RhoA and RhoC, which has been implicated in tumorigenesis, tumor progression and metastasis of $\mathrm{HCC}$ [11-15] by determining the clinical correlation of their expressions individually and in combination with HCC prognosis. Our study will demonstrate the pivotal role of Pin1 in HCC metastasis and prognosis.

\section{Methods}

\section{Patients and specimens}

Fresh tumor specimens were randomly obtained from 139 patients (79 patients with paired tumor/non-tumor specimens and 60 patients with tumor specimens only) who underwent surgical resection of primary HCC at the Department of Surgery, Queen Mary Hospital, The University of Hong Kong, between 1998 and 2007. One hundred and four were men and thirty-five were women. Patients' age ranged from 15 to 88 years, with a mean age of 58.6 years. The patient characteristics were summarized in Additional file 1: Table S1 and Additional file 2: Table S2. All samples were immediately frozen in liquid nitrogen and kept at $-80^{\circ} \mathrm{C}$ until analysis. The study was approved by Institutional Review Board of the University of Hong Kong/ Hospital Authority Hong Kong West Cluster (HKU/HA HKW IRB) and written informed consents were obtained from patients prior to their inclusion.

\section{RNA extraction and CDNA synthesis}

Total RNA was extracted from tumors and their corresponding non-tumorous liver samples (if available) from HCC patients using Trizol reagent and RNA miniprep extraction kit according to the manufacture's instruction (Life Technologies), and 500 ng each RNA sample was used to prepare cDNA using PrimeScript ${ }^{\mathrm{mm}}$ RT Master Mix (TaKaRa).

\section{Quantitative real-time polymerase chain reaction}

Real-time PCR was performed in a final volume of $15 \mu \mathrm{l}$ containing $1.5 \mu \mathrm{l}$ RT transcript, $0.2 \mu \mathrm{M}$ of each primer, $1 \mathrm{X}$ ROX reference dye and $7.5 \mu \mathrm{l}$ of FastStart Universal SYBR
Green Master (ROX) (Roche). The primers sequences were as follows: Pin1-FP: AAGATGGCGGACGAGGAG, Pin1RP: CACTCAGTGCGGAGGATGAT; RhoA-FP: GTGC CCACAGTGTTTGAG, RhoA-RP: AGGGCTGTCGATGGAAAAA; RhoC-FP: GCAGGGCAGGAAGACTAT, RhoC-RP: GTTGGGGCAGAAGTGCTT; actin-FP: 5'CGAGCATCCCCCAAAGTT-3', actin-RP: 5'-GCAC GAAGGCTCATCATT-3'. Real-time

PCR was carried out using the ABI 7900HT Fast RealTime PCR System (Applied Biosystems) at $95^{\circ} \mathrm{C}$ for 10 min, followed by 40 cycles at $95{ }^{\circ} \mathrm{C}$ for $15 \mathrm{sec}$ and at 56 ${ }^{\circ} \mathrm{C}$ for $1 \mathrm{~min}$. The expression of actin was used to normalize that of the target genes. Each assay was done in triplicate, the average was calculated, and the expression level of targets mRNA was expressed as fold to actin..

\section{Statistical analysis}

Data analysis was performed using SigmaStat 3.5 (Systat Software Inc., San Jose, CA, USA) or SPSS 16.0 (SPSS, Chicago, IL, USA). The Rank Sum test was used to analyze differences between experimental groups of clinical specimens. Spearman's correlation test was applied to determine correlations. Chi-square test was used to compare categorized data. Disease-free survival (DFS) was calculated by the Kaplan-Meier method, and differences in survival rate were compared using the log-rank test. Univariate and multivariate analyses were performed using the Cox proportional hazards regression model. $P<0.05$ was considered statistically significant.

\section{Results}

\section{Overexpression of pin 1, rho a and rho $\mathrm{C}$ in $\mathrm{HCC}$}

To study the role of Pin1, RhoA and RhoC in HCC carcinogenesis and progression, we first examined their transcript levels in 79 pairs of tumors and the adjacent non-tumorous liver of HCC patients by quantitative polymerase chain reaction, and expressed as the fold when compared with transcript level in the adjacent non-tumorous liver (Figure 1a-c). Pin1, RhoA and RhoC all showed significantly higher expression in HCC specimens $(2.05,3.58$ and 5.39- fold, respectively), suggesting that they were in general overexpressed in HCC. We categorized the HCC patients into three groups according to the tumor to non-tumorous liver fold change of each of these transcripts (Figure 1d). Pin1, RhoA and RhoC were overexpressed in 21, 25 and 30 patients out of 79 patients, respectively, indicating these transcripts were frequently overexpressed in HCC.

We next examined the correlation of tumor level of Pin1, RhoA and RhoC transcripts with the clinicopathological parameters of the HCC patients. As shown in Table 1, their expressions were not correlated with gender, HBsAg status and cirrhosis. High expressions of Pin1, RhoA and RhoC 


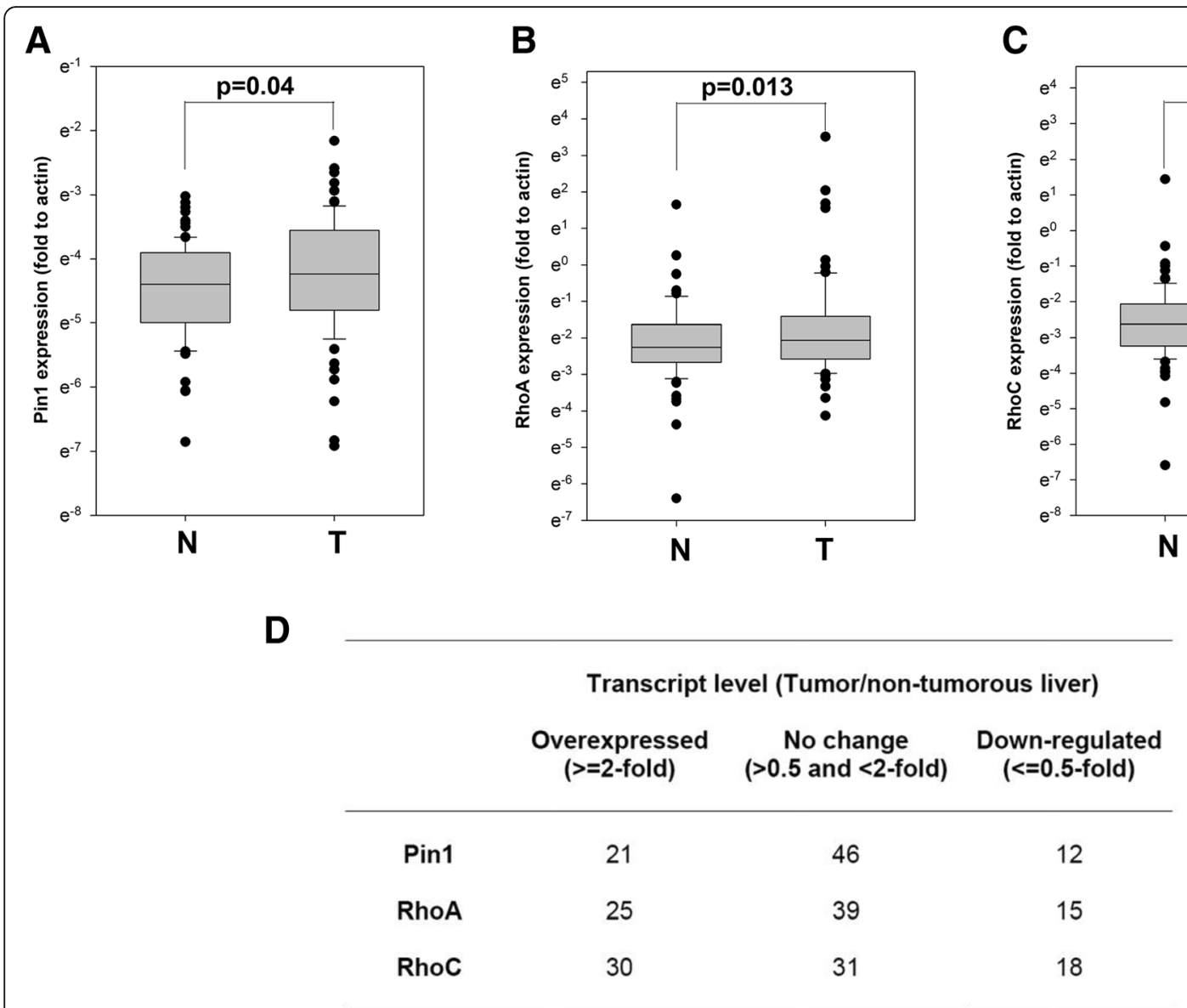

Fig. 1 Expression of Pin1, RhoA and RhoC in HCC patients. The expression of Pin1 (a), RhoA (b) and RhoC (c) in *T* representing tumor tissue and ${ }^{*} N *$ representing paired adjacent non-tumorous liver of $\mathrm{HCC}$ patients were calculated by the $2^{-\Delta \mathrm{Ct} \text { (target gene-actin) }}$ method. Results are shown as box-plot representing the entire cohort. In the box-plot (first, third quartile, median), whiskers indicate maximum/minimum values and dots indicate outliers. (d), Statistics of Pin1, RhoA and RhoC expression in HCC patients, categorized into three groups (i.e. overexpressed, no change or downregulated) according to the expression in tumor tissue when compared with the paired adjacent non-tumorous liver

were correlated with higher stage, presence of distant metastasis and recurrent disease. Interestingly, higher Pin1 and RhoC expression were significantly associated with smaller tumor, and high RhoA also demonstrated such trend, which might be due to dissemination of HCC cells from the primary tumor. Nevertheless, our clinical results demonstrated the close association of Pin1, RhoA and RhoC with metastatic HCC.

\section{Correlation between Pin1, RhoA and RhoC expressions and clinicopathologic features}

Pin1, RhoA and RhoC showed apparently higher expression in HCC with higher stage and recurrence (Table 1), we hypothesized that their co-expressions were associated with these metastatic behaviors. To test our hypothesis, we first correlated the expressions of Pin1, RhoA and RhoC in HCC patients (Figures 2a-c). The results showed that expression of Pin1 was significantly correlated with RhoA $(\mathrm{R}=0.562, \mathrm{p}<0.001)$ and $\mathrm{RhoC}(\mathrm{R}=$
0.529, $\mathrm{p}<0.001)$, whereas RhoA and RhoC showed an even stronger correlation $(R=0.950, p<0.001)$. Their close correlations supported our proposition that Pin1 associated with HCC metastasis through the action of RhoA and RhoC.

We next examined the association of Pin1, RhoA and RhoC co-expressions with stage. Overexpression of at least one of them were significantly correlated with higher stage (Table 2), and the association was stronger than that observed with Pin1, RhoA or RhoC alone as indicated by the lower p-value. In addition, Pin 1 and RhoA/RhoC cooverexpressions were significantly associated with metastasis $(\mathrm{p}=0.000012)$ and the association was again stronger than individual overexpression of Pin1, RhoA or RhoC (Table 3). Furthermore, their co-overexpression revealed significant correlation with recurrence $(\mathrm{p}<0.00001)$ when compared with overexpression of Pin $1 \quad(\mathrm{p}=0.000135)$, RhoA ( $\mathrm{p}=0.000044)$ or RhoC $(\mathrm{p}=0.00002)$ alone (Table 4$)$. These results demonstrated that co-overexpressions of 
Table 1 Correlation between Pin1, RhoA and RhoC expression and clinicopathological parameters of the HCC patients in this study The median, lower quartile and upper quartile of the relative expression of each gene (fold to actin) of different experimental group were shown. Rank Sum test was performed to compare the difference

\begin{tabular}{|c|c|c|c|}
\hline Gender & Male & Female & $p$-value \\
\hline Pin1 & $0.039(0.004-0.074)$ & $0.023(0.001-0.095)$ & 0.317 \\
\hline RhoA & $0.558(0.101-1.264)$ & $0.479(0.121-1.675)$ & 0.871 \\
\hline RhoC & $0.182(0.066-0.677)$ & $0.314(0.096-0.751)$ & 0.364 \\
\hline Age & $\leq 55$ & $>55$ & $p$-value \\
\hline Pin1 & $0.004(0.000-0.048)$ & $0.058(0.005-0.151)$ & $<0.001$ \\
\hline RhoA & $0.189(0.076-0.617)$ & $0.941(0.167-2.518)$ & $<0.001$ \\
\hline RhoC & $0.141(0.056-0.355)$ & $0.460(0.1290 .897$ & $<0.001$ \\
\hline $\mathrm{HBsAg}$ & negative & positive & $p$-value \\
\hline Pin1 & $0.058(0.002-0.095)$ & $0.020(0.001-0.096)$ & 0.284 \\
\hline RhoA & $0.680(0.121-1.936)$ & $0.408(0.117-1.567)$ & 0.670 \\
\hline RhoC & $0.356(0.112-0.737)$ & $0.255(0.090-0.774)$ & 0.966 \\
\hline Cirrhosis & negative & positive & $p$-value \\
\hline Pin1 & $0.011(0.001-0.075)$ & $0.044(0.003-0.096)$ & 0.143 \\
\hline RhoA & $0.346(0.103-1.433)$ & $0.790(0.126-1.807)$ & 0.308 \\
\hline RhoC & $0.238(0.095-0.760)$ & $0.383(0.086-0.654)$ & 0.678 \\
\hline Tumor size & $\leq 5$ & $>5$ & $p$-value \\
\hline Pin1 & $0.045(0.006-0.113)$ & $0.008(0.001-0.074)$ & 0.021 \\
\hline RhoA & $0.913(0.189-1.654)$ & $0.245(0.099-1.354)$ & 0.061 \\
\hline RhoC & $0.433(0.165-0.778)$ & $0.187(0.080-0.627)$ & 0.048 \\
\hline Stage & low & high & $p$-value \\
\hline Pin1 & $0.006(0.001-0.061)$ & $0.060(0.018-0.152)$ & $<0.001$ \\
\hline RhoA & $0.217(0.101-0.944)$ & $1.029(0.306-2.750)$ & 0.002 \\
\hline RhoC & $0.187(0.082-0.532)$ & $0.470(0.187-1.259)$ & 0.003 \\
\hline Metastasis & absent & present & $p$-value \\
\hline Pin1 & $0.006(0.008-0.065)$ & $0.065(0.048-0.260)$ & $<0.001$ \\
\hline RhoA & $0.210(0.096-0.984)$ & $1.746(0.926-4.374)$ & $<0.001$ \\
\hline RhoC & $0.188(0.073-0.499)$ & $0.763(0.371-1.880)$ & $<0.001$ \\
\hline Recurrence & absent & present & $p$-value \\
\hline Pin1 & $0.008(0.001-0.051)$ & $0.058(0.003-0.150)$ & 0.003 \\
\hline RhoA & $0.189(0.094-0.758)$ & $1.137(0.306-3.301)$ & $<0.001$ \\
\hline RhoC & $0.132(0.064-0.436)$ & $0.501(0.207-1.377)$ & $<0.001$ \\
\hline
\end{tabular}

Pin1, RhoA/RhoC were crucial factors for HCC metastasis and recurrence.

\section{Effect of Pin1/RhoA/RhoC expression on recurrence-free survival}

In order to identify patients with higher potential to develop future distant metastasis, we compared the prognosis of HCC patients with and without overexpression of Pin1, RhoA or RhoC, as well as those with and without co-overexpression of these three genes. As shown in
Figures 2d-g, downregulation of Pin1, RhoA or RhoC displayed signficantly better recurrence-free survival. Moreover, patients with co-overexpressions of Pin1, RhoA and RhoC showed a significantly poor recurrencefree survival $(\mathrm{p}<0.001)$..

A univariate Cox regression analysis was applied to identify important prognostic factors of recurrence-free survival, which tested parameters including age, gender, HBsAg status, cirrhosis, tumor size, TNM stage and Pin1/RhoA/RhoC co-expressions (Table 5). High stage and co-overexpressions of Pin $1+$ RhoA/RhoC were identified as important risk factors for recurrence-free survival $(\mathrm{p}<0.001)$. Next, we generated a multivariate analysis using Cox regression model, which againshowed that higher stageand no co-overexpressions of Pin1+RhoA/RhoC were independent factors for the recurrence-free survival of $\mathrm{HCC}$ patients $(\mathrm{p}<0.001$, Table 5). These results demonstrated that co-overexpressions of Pin $1+$ RhoA/RhoC was an unfavorable prognostic factor for recurrence-free survival of HCC patients, and potentiated these genes as molecular therapeutic targets to combat HCC metastasis.

\section{Discussion}

Development of metastasis accounts for the extremely poor prognosis of HCC patients. Therefore, an important part of HCC researches is the identification of crucial factors for HCC metastasis, which are potential biomarkers to predict the prognosis of patients, or even novel therapeutic target to combat HCC metastasis. Our team and other groups have previously reported the roles of Pin1 in HCC carcinogenesis and its clinical correlation with $\mathrm{HCC}$ oncogenesis (6-10), yet its association with metastasis has not been demonstrated in HCC thus far. On the other hand, RhoA and RhoC overexpressions have been correlated with HCC progression and metastasis in many clinical studies [11-16]. In this study, we aim to examine the correlation between HCC metastasis and expressions of Pin1, RhoA and RhoC, and further determine the clinical significance of their coexpressions.

This study demonstrates for the first time the correlation of Pin1, RhoA and RhoC with the development of metastasis in HCC patients. We found that HCC patients with overexpression of Pin1, RhoA or RhoC showed increased rate of metastasis, when compared with those with down-regulation counterparts. For example, around $40 \%$ of HCC patients with overexpression of Pin1, RhoA or RhoC developed distant metastasis, whereas the percentage decreased to around $10 \%$ in patients with down-regulation of Pin1, RhoA or RhoC, suggesting that their down-regulations impaired the metastatic processes. In addition, we further demonstrated that $46 \%$ of the patients with co-overexpressions 


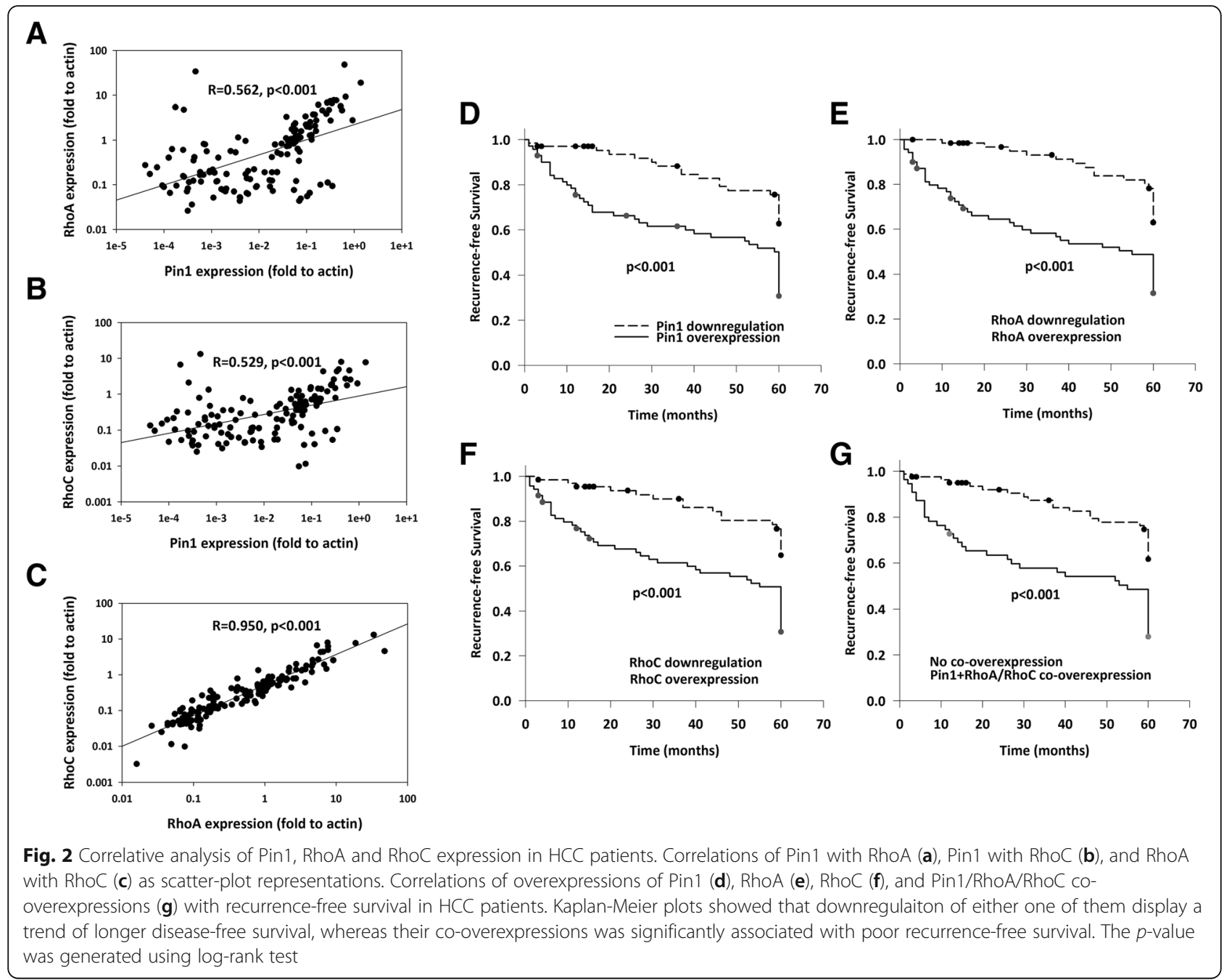

Table 2 Clinical correlation of Pin1, RhoA and RhoC expressions with stage of HCC patients using Fisher exact test

\begin{tabular}{llll}
\hline & High (III-IV) & Low (I-II) & $p$-value \\
\hline Pin1 expression & 36 & 36 & \\
Overexpression & 14 & 14 & 0.000094 \\
Downregulation & & & \\
RhoA expression & 33 & 32 & \\
Overexpression & 17 & 47 & 0.00478 \\
Downregulation & & & \\
RhoC expression & 34 & 31 & \\
Overexpression & 16 & 48 & 0.001458 \\
Downregulation & & & \\
Pin1/RhoA/RhoC expression & & 39 & \\
At least 1 overexpression & 43 & 40 & 0.000025 \\
All downregulation & 7 & &
\end{tabular}

Table 3 Clinical correlation of Pin 1, RhoA and RhoC expressions with HCC metastasis using Fisher exact test

\begin{tabular}{llll}
\hline & $\begin{array}{l}\text { High } \\
\text { (III-IV) }\end{array}$ & $\begin{array}{l}\text { Low } \\
(I-I I)\end{array}$ & $p$-value \\
\hline
\end{tabular}

Pin1 expression

Overexpression $\quad 28 \quad 42$

Downregulation $\quad 6 \quad 62$

0.000021

RhoA expression

$\begin{array}{lll}\text { Overexpression } & 27 & 41 \\ \text { Downregulation } & 7 & 63\end{array}$

RhoC expression

Overexpression $\quad 27 \quad 42$

Downregulation

$7 \quad 61$

0.000094

Pin1 + RhoA/RhoC co-expressions

Co-overexpression of Pin 1 + RhoA/RhoC $26 \quad 31$

\begin{tabular}{llll} 
No co-overexpression & 8 & 73 & 0.000012 \\
\hline
\end{tabular} 
Table 4 Clinical correlation of Pin1, RhoA and RhoC expressions with recurrence of HCC patients using Fisher exact test

\begin{tabular}{llll}
\hline & Presence & Absence & $p$-value \\
\hline Pin1 expression & & & \\
$\quad$ Overexpression & 44 & 22 & \\
$\begin{array}{l}\text { Downregulation } \\
\text { RhoA expression }\end{array}$ & 25 & 48 & 0.000135 \\
$\quad$ Overexpression & 44 & 21 & \\
$\quad$ Downregulation & 24 & 49 & 0.000044 \\
RhoC expression & & & \\
$\quad$ Overexpression & 45 & 20 & \\
$\quad$ Downregulation & 24 & 49 & 0.00002 \\
Pin1+RhoA/RhoC co-expressions & & & \\
$\quad \begin{array}{l}\text { Co-overexpression of Pin1 + RhoA/ } \\
\text { RhoC }\end{array}$ & 43 & 21 & \\
No co-overexpression & 18 & 55 & $<0.00001$ \\
\hline
\end{tabular}

of Pin $1+$ RhoA/RhoC developed distant metastasis, while less than $10 \%$ patients with no such co-overexpressions developed metastasis. Similar trends were also observed in the recurrence-free survival analyses of HCC patients, in which patients with downregulations of Pin1, RhoA or RhoC showed a trend of better prognosis for recurrence-free survival when compared with those with overexpressions, and those with co-overexpressions of Pin1+RhoA/RhoC demonstrated a significantly poor recurrence-free survival than those with no co-overexpressions. Hence, our results clearly demonstrated the correlations of Pin1 with RhoA and RhoC as well as their involvement in the metastatic process, and their co-overexpressions associated with metastatic HCC, which further potentiates the development of combined inhibitors against Pin1/RhoA/RhoC in order to combat metastasis in HCC patients.

There are two possible linkages between Pin1 and RhoA/ RhoC. Our previous study on Pin1 demonstrated a preferential Pin1 overexpression in HBV-related tumors [9], whereas other RhoC studies showed that HBV proteins including $\mathrm{HBx}$ and $\mathrm{HBs}$ enhanced the promoter activity of RhoC in vitro through upregulating the transcription factor Ets-1, resulting in overexpression of RhoC mRNA and proteins $[17,18]$. In accordance, our results in this report revealed that $\mathrm{HCC}$ patients with $\mathrm{HBsAg}$ showed a significantly stronger overexpression of Pin1 and RhoC, and similar trend for RhoA, in HCC when compared to adjacent normal liver (Additional file 3: Figure S1), though the effect of $\mathrm{HBV}$ proteins on their transcription has not been demonstrated yet. Moreover, we previously demonstrated that interaction and binding of Pin 1 to phosphorylated Ser41Pro motif of $\mathrm{HBx}$, followed by cis-trans isomerization and stabilization, significantly augmented the expression of $\mathrm{HBx}$ downstream target genes [9]. Hence we believed that Pin 1 could regulate the expression of RhoA/RhoC through this pathway, as we observed a strong correlation between Pin1 and RhoA/RhoC expressions. In addition to upregulation of the RhoA/RhoC transcription level, Pin1 is also likely to affect the activity of RhoA/RhoC indirectly through its effect on $\mathrm{HBx}$ protein which activates

Table 5 Univariate and multivariate analyses showing that Pin1/RhoA/RhoC co- overexpression could serve as an independent prognostic factor for recurrence of HCC patients

\begin{tabular}{|c|c|c|}
\hline & Relative risk ( $95 \%$ confidence interval & $P$-value \\
\hline \multicolumn{3}{|l|}{ Univariate analysis } \\
\hline Age, year (> 55 versus $\leq 55$ ) & $1.350(0.802-2.270)$ & 0.258 \\
\hline Gender (male versus female) & $1.041(0.576-1.881)$ & 0.894 \\
\hline HBsAg (positive versus negative) & $0.820(0.451-1.489)$ & 0.513 \\
\hline Cirrhosis (yes versus no) & $0.921(0.565-1.503)$ & 0.743 \\
\hline Tumor Size (> 5 versus $\leq 5$ ) & $1.576(0.950-2.614)$ & 0.078 \\
\hline TNM stage (III to IV versus I to II) & $4.857(2.841-8.303)$ & $<0.001$ \\
\hline Pin1 + RhoA/RhoC co-overexpression (yes versus no) & $2.804(1.686-4.663)$ & $<0.001$ \\
\hline \multicolumn{3}{|l|}{ Multivariate analysis } \\
\hline Age, year (> 55 versus $\leq 55$ ) & $0.749(0.3359-1.564)$ & 0.442 \\
\hline Gender (male versus female) & $0.838(0.433-1.622)$ & 0.600 \\
\hline HBsAg (positive versus negative) & $0.958(0.495-1.854)$ & 0.899 \\
\hline Cirrhosis (yes versus no) & $0.890(0.498-1.590)$ & 0.694 \\
\hline Tumor Size ( $>5$ versus $\leq 5$ ) & $1.268(0.692-2.326)$ & 0.442 \\
\hline TNM stage (III to IV versus I to II) & $3.453(1.814-6.573)$ & $<0.001$ \\
\hline Pin1 + RhoA/RhoC co-overexpression (yes versus no) & $2.159(1.078-4.325)$ & $<0.001$ \\
\hline
\end{tabular}


RhoA in vitro [19], as well as directly or indirectly interacts with RhoA and $\mathrm{RhoC}$ and regulates activation, as we had confirmed the presence of interaction between Pin 1 and RhoA/RhoC in our coimmunoprecipitation experiment (our unpublished results). Further experiments are warranted and still in progress to investigate the effect of Pin1 on the functional roles and molecular mechanism of RhoA and $\mathrm{RhoC}$ in HCC metastasis in vitro and in vivo.

In this study, we showed that the combination of biomarkers demonstrated a better prognostic value than either one of Pin1, RhoA or RhoC, suggesting that these factors act together in the cancer metastatic process and thus effect of downregulation of one factor could be compensated by the others. Therefore, it will be more comprehensive to monitor a combination of factors in this instance. Indeed, there are increasing evidences demonstrating the efficacy of combination of molecularly-associated biomarkers. For example, combination of osteopontin and its receptor CD44v6 improves the sensitivity and predictive range for predicting tumor recurrence and survival in non-small cell lung cancer patients [20], combination of hypoxia-induced autophagy- related gene Beclin1 and HIF- $1 \alpha$ refine distant metastasis risk and predict poor prognosis of ER-positive, HER2-negative breast cancer [21], and miR-34a silencing in combination with its target genes c-Met and $\beta$-catenin predicts distant metastasis of colon cancer [22]. The results obtained in this and the above studies, demonstrated that a set of markers provided a better monitoring of the tumor cellular process which was associated with a more precise prognosis of the tumor condition, as well as identified potential targets to inhibit the progression of tumor or development of metastasis.

\section{Conclusion}

To summarize, this study demonstrated for the first time the clinical correlation of Pin1 in HCC metastasis. Pin1 was significantly correlated with the transcript levels of RhoA and $\mathrm{RhoC}$, and their co-overexpressions correlated with metastatic HCC and recurrence of HCC patients, suggesting that Pin1, RhoA and RhoC not only could be used as potential biomarker for predicting development of metastasis after curative resection, but also as potential therapeutic targets to inhibit HCC metastasis.

\section{Additional files}

Additional file 1: Table S1. Patient characteristics in this study. (PDF 30 $\mathrm{kb})$

Additional file 2: Table S2. Pin1, RhoA and RhoC expressions (fold to actin) in all HCC samples and the patient's clinicopathological parameters. (XLSX $25 \mathrm{~kb}$ )
Additional file 3: Figure S1. Comparison of Pin1, RhoA and RhoC overexpressions (Tumor/non-tumor) in HCC patients with or without HBsAg. (TIF $2557 \mathrm{~kb}$ )

\section{Abbreviations}

HCC: Hepatocellular carcinoma; N: Adjacent non-tumor liver; Pin 1: Peptidylprolyl cis-trans isomerase NIMA-interacting 1; RhoA: Ras homolog gene

family, member A; RhoC: Ras homolog gene family, member C; T: Tumor

\section{Acknowledgements}

We would like to thank Miss Tracy LAU and the Centre for Cancer Research of the University of Hong Kong for providing technical support and equipment for this study.

\section{Authors' contributions}

LN performed the experiments, analyzed the data and write the manuscript. VK, AC performed the experiments. TCCY, RTPP, RP and WLL analyzed and interpreted the patient data. All authors read and approved the final manuscript.

\section{Funding}

This work is supported by the Research Grant Council, Hong Kong (HKU$774309 \mathrm{M})$. The funding bodies had no role in the design of the study and collection, analysis, and interpretation of data and in writing the manuscript.

Availability of data and materials

All data generated or analyzed during this study are included in this published article [and its supplementary information files].

\section{Ethics approval and consent to participate}

The study was approved by Institutional Review Board of the University of Hong Kong/Hospital Authority Hong Kong West Cluster (HKU/HA HKW IRB) and written informed consents were obtained from patients prior to their inclusion.

Consent for publication

Not applicable.

Competing interests

The authors declare that they have no competing interests.

\section{Author details}

${ }^{1}$ Department of Surgery, Li Ka Shing Faculty of Medicine, The University of Hong Kong, Pok Fu Lam, Hong Kong. ${ }^{2}$ Centre for Cancer Research, Li Ka Shing Faculty of Medicine, The University of Hong Kong, Pok Fu Lam, Hong Kong. ${ }^{3}$ Department of Medicine, Li Ka Shing Faculty of Medicine, The University of Hong Kong, Pok Fu Lam, Hong Kong.

Received: 16 December 2018 Accepted: 10 July 2019

Published online: 19 July 2019

\section{References}

1. Wong MC, Jiang JY, Goggins WB, Liang M, Fang Y, Fung FD, Leung C, Wang $\mathrm{HH}$, Wong GL, Wong WW, et al. International incidence and mortality trends of liver cancer: a global profile. Sci Rep. 2017;7:45846.

2. Llovet JM, Schwartz M, Mazzaferro V. Resection and liver transplantation for hepatocellular carcinoma. Semin Liver Dis. 2005;25(2):181-200.

3. Lu KP, Hanes SD, Hunter T. A human peptidyl-prolyl isomerase essential for regulation of mitosis. Nature. 1996;380(6574):544-7.

4. Ranganathan R, Lu KP, Hunter T, Noel JP. Structural and functional analysis of the mitotic rotamase Pin 1 suggests substrate recognition is phosphorylation dependent. Cell. 1997:89(6):875-86.

5. Yaffe MB, Schutkowski M, Shen M, Zhou XZ, Stukenberg PT, Rahfeld JU, Xu J, Kuang J, Kirschner MW, Fischer G, et al. Sequence-specific and phosphorylation-dependent proline isomerization: a potential mitotic regulatory mechanism. Science. 1997;278(5345):1957-60.

6. Pang R, Yuen J, Yuen MF, Lai CL, Lee TK, Man K, Poon RT, Fan ST, Wong CM, $\mathrm{Ng}$ IO, et al. PIN1 overexpression and beta-catenin gene mutations are distinct oncogenic events in human hepatocellular carcinoma. Oncogene. 2004;23(23):4182-6. 
7. Cheng CW, Chow AK, Pang R, Fok EW, Kwong YL, Tse E. PIN1 inhibits apoptosis in hepatocellular carcinoma through modulation of the antiapoptotic function of survivin. Am J Pathol. 2013;182(3):765-75.

8. Lee NY, Choi HK, Shim JH, Kang KW, Dong Z, Choi HS. The prolyl isomerase Pin1 interacts with a ribosomal protein S6 kinase to enhance insulin-induced AP-1 activity and cellular transformation. Carcinogenesis. 2009;30(4):671-81.

9. Pang R, Lee TK, Poon RT, Fan ST, Wong KB, Kwong YL, Tse E. Pin1 interacts with a specific serine-proline motif of hepatitis $B$ virus $X$-protein to enhance hepatocarcinogenesis. Gastroenterology. 2007;132(3):1088-103.

10. Pang RW, Lee TK, Man K, Poon RT, Fan ST, Kwong YL, Tse E. PIN1 expression contributes to hepatic carcinogenesis. J Pathol. 2006;210(1):19-25.

11. Hu T, Guo H, Wang W, Yu S, Han L, Jiang L, Ma J, Yang C, Guo Q, Nan K. Loss of p57 expression and RhoA overexpression are associated with poor survival of patients with hepatocellular carcinoma. Oncol Rep. 2013;30(4):1707-14.

12. Gou L, Wang W, Tong A, Yao Y, Zhou Y, Yi C, Yang J. Proteomic identification of RhoA as a potential biomarker for proliferation and metastasis in hepatocellular carcinoma. J Mol Med (Berl). 2011;89(8):817-27.

13. Wang D, Dou K, Xiang H, Song Z, Zhao Q, Chen Y, Li Y. Involvement of RhoA in progression of human hepatocellular carcinoma. J Gastroenterol Hepatol. 2007;22(11):1916-20.

14. Wang W, Yang LY, Huang GW, Lu WQ, Yang ZL, Yang JQ, Liu HL. Genomic analysis reveals RhoC as a potential marker in hepatocellular carcinoma with poor prognosis. Br J Cancer. 2004;90(12):2349-55.

15. Wang W, Yang LY, Yang ZL, Huang GW, Lu WQ. Expression and significance of RhoC gene in hepatocellular carcinoma. World J Gastroenterol. 2003:9(9):1950-3.

16. Li XR, Ji F, Ouyang J, Wu W, Qian LY, Yang KY. Overexpression of RhoA is associated with poor prognosis in hepatocellular carcinoma. Eur J Surg Oncol. 2006;32(10):1130-4.

17. Tian Y, Liu Y, Qu J, Li K, Qin D, Huang A, Tang H. HBV regulated RhoC expression in HepG2.2.15 cells by enhancing its promoter activity. J Basic Microbiol. 2013;53(5):461-8.

18. Qin D, Li K, Qu J, Wang S, Zou C, Sheng Y, Huang A, Tang H. HBx and HBs regulate RhoC expression by upregulating transcription factor Ets-1. Arch Virol. 2013;158(8):1773-81.

19. Feng $H$, Li X, Niu D, Ning Chen W. HBx expression activates RhoA GTPase: impact on cell migration. Cell Biol Int. 2011;35(2):159-64.

20. Sun BS, Li Y, Zhang ZF, You J, Wang CL. Osteopontin combined with CD44v6, a novel prognostic biomarker in non-small cell lung cancer undergoing curative resection. Ann Thorac Surg. 2013;96(6):1943-51.

21. Dong $M$, Wan XB, Yuan ZY, Wei L, Fan XJ, Wang TT, LV YC, Li X, Chen $\mathrm{ZH}$, Chen J, et al. Low expression of Beclin 1 and elevated expression of HIF-1alpha refine distant metastasis risk and predict poor prognosis of ER-positive, HER2-negative breast cancer. Med Oncol. 2013;30(1):355.

22. Siemens H, Neumann J, Jackstadt R, Mansmann U, Horst D, Kirchner T, Hermeking $\mathrm{H}$. Detection of miR-34a promoter methylation in combination with elevated expression of c-met and beta-catenin predicts distant metastasis of colon cancer. Clin Cancer Res. 2013;19(3):710-20.

\section{Publisher's Note}

Springer Nature remains neutral with regard to jurisdictional claims in published maps and institutional affiliations.

Ready to submit your research? Choose BMC and benefit from:
- fast, convenient online submission
- thorough peer review by experienced researchers in your field
- rapid publication on acceptance
- support for research data, including large and complex data types
- gold Open Access which fosters wider collaboration and increased citations
- maximum visibility for your research: over 100M website views per year
At BMC, research is always in progress.
Learn more biomedcentral.com/submissions

